Document downloaded from:

http://hdl.handle.net/10251/66809

This paper must be cited as:

Elena Fito, SF.; Carrera, J.; Rodrigo, J. (2011). A systems biology approach to the evolution of plant-virus interactions. Current Opinion in Plant Biology. 14(4):372-377. doi:10.1016/j.pbi.2011.03.013.

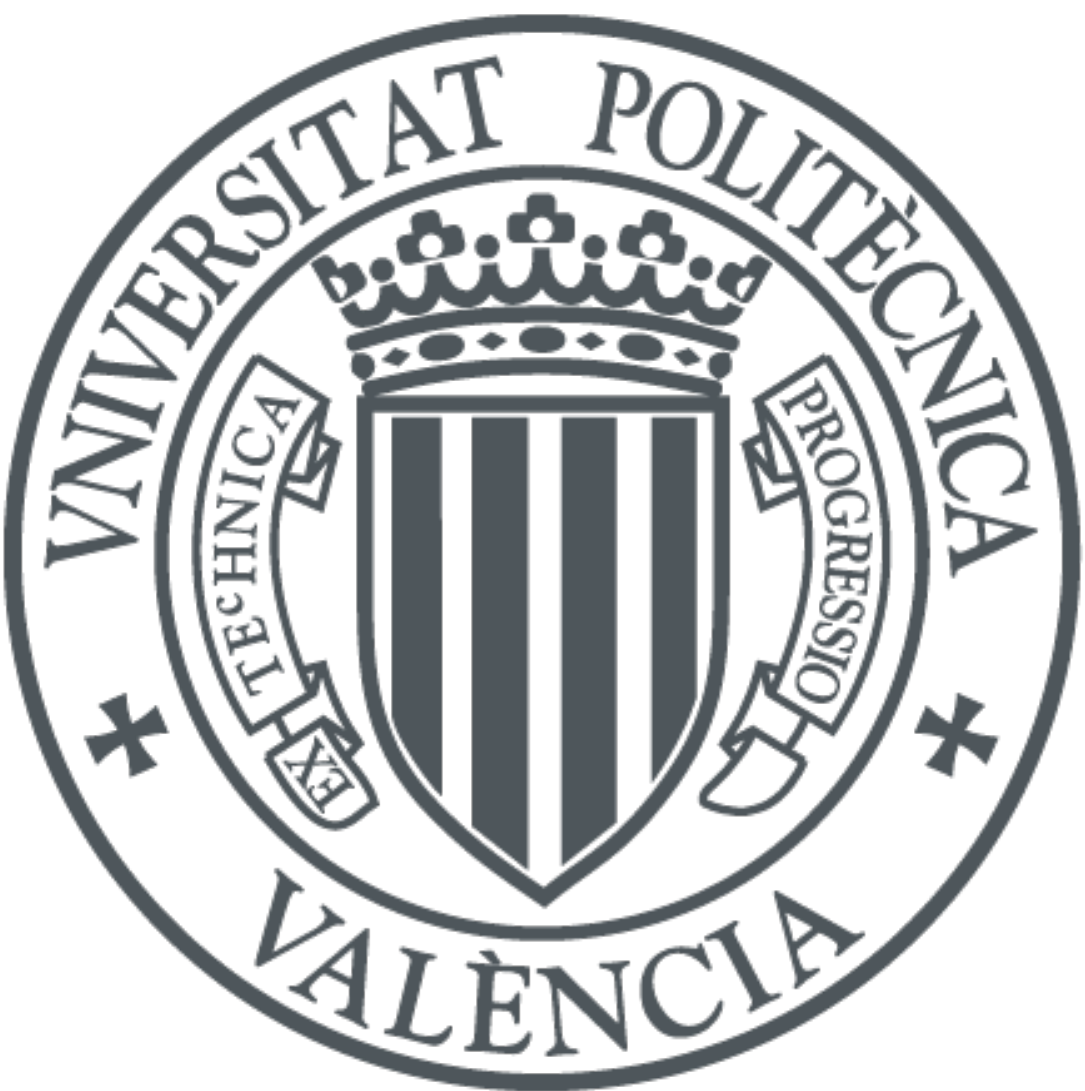

The final publication is available at

http://dx.doi.org/10.1016/j.pbi.2011.03.013

Copyright Elsevier

Additional Information 


\title{
A systems biology approach to the evolution of plant-virus interactions
}

\author{
Santiago F. Elena ${ }^{1,2}$, Javier Carrera ${ }^{1,3}$, and Guillermo Rodrigo ${ }^{1}$ \\ ${ }^{1}$ Instituto de Biología Molecular y Celular de Plantas, CSIC-UPV, Ingeniero Fausto Elio s/n, \\ 46022 València, Spain \\ ${ }^{2}$ The Santa Fe Institute, 1399 Hyde Road Park, Santa Fe, NM 87501, USA \\ ${ }^{3}$ Instituto ITACA, UPV, Ingeniero Fausto Elio s/n, 46022 València, Spain
}

\section{Corresponding author: Santiago F. Elena}

Omic approaches to the analysis of plant-virus interactions are becoming increasingly popular. These types of data, in combination with models of interaction networks, will aid in revealing not only host components that are important for the virus life cycle, but also general patterns about the way in which different viruses manipulate host regulation of gene expression for their own benefit and possible mechanisms by which viruses evade host defenses. Here, we review studies identifying host genes regulated by viruses and discuss how these genes integrate in host regulatory and interaction networks, with a particular focus on the physical properties of these networks.

\section{The systems biology approach}

Genomic tools have allowed assessment of gene expression at a genome-wide scale, providing unprecedented views of the host-virus interaction. To make use of all of the information contained in these large data sets, however, it is necessary to use computational and mathematical tools to disentangle the interactions between the molecular components of both biological entities and to identify how these interactions determine the outcome of the infection $[1,2 \bullet \bullet]$, which is known as the field of genomic systems biology (GSB). GSB is a top-down approach that takes advantage of the recent development of high-throughput experimental techniques for obtaining omic data, and constitutes the antithesis of the reductionist paradigm (with a bottom-up perspective) that has been dominating molecular biology. The GSB approach consists of cycling between the generation of experimental data and modeling by means of reverse-engineering techniques to propose testable hypotheses about biological systems, experimental validation of these hypotheses and quantification of the relevant model parameters, and then using the newly acquired quantitative description to refine the computational model and finally make predictions of the system behavior $[3,4 \bullet]$.

To complete its infectious cycle, a few components of a virus, including its nucleic acids and encoded proteins, must establish multiple and complex interactions not only among themselves $[5,6 \bullet, 7,8]$ but also with a myriad of components of the host cell $[9,10 \bullet, 11]$. The outcome of all these interactions is that the plant controls the spread of viral infection or, alternatively, the virus overcomes the host defenses and establishes a productive infection that may or may not be associated with the development of symptoms. While the GSB approach is being extensively used in the analysis of animal virus interactions (e.g. hepatitis $\mathrm{C}$, human immunodeficiency, yellow fever, influenza A and herpesviruses), plant virology has not yet benefitted to the same extent, and the most relevant studies in the field generally apply some transcriptomics techniques to produce lists of genes with altered mRNA 
abundance in infected plants relative to controls. However, these types of studies still produce very useful data and serve to highlight some interesting features. Indeed, recent application of the GSB approach to the analysis of animal-virus interactions has revealed new and interesting insights. For example, thoughtful statistical analyses of expression data has facilitated identification of virus-regulated genes (VRGs), some of which encode cellular factors required for completion of the infection cycle, while others are direct targets that the virus manipulates to deactivate the cell defense mechanisms $[9,12-14]$. It has also been observed that VRGs are preferentially highly connected elements in the host regulatory network $[15,16,17 \bullet \bullet]$. Furthermore, it has been observed that the topological properties of the intraviral interaction network change as a consequence of its integration within the host network $[6 \bullet, 18]$. In the next section we explore how many of these findings have been extended to plant viruses.

\section{Compiling and comparing expression data for several plant viruses}

Although some studies have analyzed changes in global profiling resulting from virus infection of natural hosts, such as infection of cassava by African cassava mosaic virus [19] and infection of rice by rice yellow mottle virus [20], Arabidopsis thaliana has been the main model host used in combination with viruses belonging to different taxonomic families. These studies involved cauliflower mosaic caulimovirus (CaMV) [21]; turnip vein clearing (TVCV) [22•], oilseed rape mosaic (OMRV) [22•] and tobacco mosaic (TMV) tobamoviruses [23,24]; potato X potexvirus (PVX) [22•]; cucumber mosaic cucumovirus (CMV) $[22 \bullet, 25,26]$; turnip mosaic (TuMV) [22 $\bullet, 27]$, plum pox (PPV) [28] and tobacco etch (TEV) potyviruses [29]; and mung bean yellow mosaic (MYMV) [30] and cabbage leaf curl $(\mathrm{CaLCuV})$ geminiviruses [31]. However, even using the same host species, direct comparisons across experiments are not straightforward because differences in profiling techniques and platforms, plant ecotypes, sampling schemes, inoculation conditions and dosages, and growth environmental variables may all exert unpredictable effects on the expression pattern of multiple genes. Furthermore, differences in statistical normalization methods and analyses also contribute to making comparisons difficult.

Whitham and coworkers [22•] carried out the most comprehensive of such studies for five viruses (CMV, ORMV, PVX, TVCV, and TuMV) while keeping constant all other experimental variables and techniques. Some generalities can be drawn from this study that can be extended to most of the other studies cited above, highlighting the fact that different viruses alter common sets of genes or biological functions (summarized in Figure 1). On the one hand, approximately one-third of overexpressed VRGs are associated with cell rescue, defense, apoptosis and cell death and aging, including several defense- and stress-associated genes. Responses to biotic (viruses, bacteria, or fungi) and abiotic (metal ions, osmosis, oxidation, or temperature) stresses, including systemic acquired resistance and the innate immune system, are upregulated by the plant to counteract viral infection. Such a defense response in $A$. thaliana to viruses is dependent on salicylic acid [32]. In addition, a variety of heat-shock proteins are also overexpressed after infection with any viruses. Although this might just be a generic nonspecific response by the plant to stress, we suggest that the virus directly triggers chaperones to assist in correct folding of its own proteins, since many of them could misfold (and thus aggregate) as a consequence of mutations produced during error-prone replication [33]. Ribosomal proteins and protein turnover genes are also upregulated. Again, this could either reflect an increased demand on the host cells for protein synthesis or a response triggered by a virus to enhance its own production (or presumably both). On the other hand, several developmental functions, biosynthesis of lipids, alcohols and polysaccharides, and secondary metabolism constitute the principal downregulated processes. For example, biosynthesis of lipids is pivotal for cell membrane construction and 
modification and carbohydrates biosynthesis is essential for building cell walls; therefore, because this expression is correlated to plant cell growth and expansion, reduced expression could well result in the stunting syndrome associated with some infections. Similarly, plastid genes and genes involved in chloroplast functioning are also preferentially underexpressed, resulting in chlorosis.

\section{Viruses preferentially alter highly connected genes}

A better understanding of the role of VRGs during infection can be drawn from an analysis of their context in the interaction network in which they exist [34,35]. Application of some basic concepts of networks theory can elucidate which of two possible orthogonal scenarios better describe VRGs: on the one hand, VRGs are essential elements with a high number of links in the interactome (i.e., hub genes) or, on the other hand, VRGs are elements randomly and sparsely distributed across the interactome network and are thus poorly connected. Figure $2 \mathrm{a}$ illustrates the second scenario whereby most VRGs have a low number of connections (connectivity degree). Comparison of the slope of the expected power-law distribution fitted to the data reveals that the whole interactome has a steeper slope than the VRG subset. Conversely, Figure $2 \mathrm{~b}$ shows a case in which VRGs are highly connected genes and thus the slope of the power-law is flatter than for the whole interactome. These predictions have been already tested for several animal RNA viruses, and the results have shown that VRGs represent subsets of highly connected genes $[15,16,17 \bullet \bullet]$. Similar studies are still lacking for plant viruses, including large-scale yeast two-hybrid assays to reveal the direct targets of viral RNAs and proteins. To that end, recent studies have proposed models for the A. thaliana protein-protein interactions network (PPIN), predicted from an analysis of interacting orthologs in the proteome of several organisms [36], and the transcriptional regulatory network (TRN), inferred from high-throughput data for the plant transcriptome $[37,38]$. These models represent the starting point for placing VRGs in the correct network context. In a unpublished study, we investigated the interactomic contextualization of different lists of VRGs to determine whether plant viruses show a general pattern of infection. Interestingly, topological analysis of VRGs shows that viruses alter the expression of master transcription factors and hub proteins, calculated from the above $A$. thaliana PPIN and TRN (Table 1).

\section{Evolution and host adaptation}

The actual interaction between viruses and their natural hosts and vectors is the result of natural selection operating over many generations. The evolutionary race between plant defenses and virus counter defenses determines whether infection results in strong symptoms with high viral production, in asymptomatic productive infection, or in failed infection. Hence, for a biologically meaningful description of the interactions established between viral and cellular components, it is necessary to take into account the degree of adaptation of the virus to the host. Unfortunately, this evolutionary aspect has not been taken into consideration in most of the studies mentioned above. CaLCuV, MYMV, ORMV, PPV, PVX, TEV, TMV, and TVCV infections of wild populations of $A$. thaliana have not been described, although $\mathrm{CaLCuV}$ and TVCV naturally infect other plants of the Brassicaceae family. Only TuMV, CMV and CaMV are prevalent in wild $A$. thaliana populations [40]. Therefore, we should be cautious when drawing strong conclusions about interactions from artificial plant-virus pathosystems unless we analyze the conserved response of plants to any infection or their nonspecific responses to biotic stresses. To illustrate whether adapted and non-adapted viruses differ in the topological properties of VRG subnetworks, Table 1 lists the number of VRGs, the number of interactions and the average degree of connectivity for these subnetworks contextualized in the $A$. thaliana PPIN and TRN. We distinguished between viruses that naturally infect brassicas (an isolate of TEV experimentally adapted to 
A. thaliana [39•], TuMV, TCV, and CaLCuV) and those that do not (TRV, TMV, PPV, and TEV), and found significant differences ( 1 -tailed $P<0.05)$ for both network models. The first set of viruses has, on average, more VRGs that are more densely connected (irrespective of the network model used), probably because of virus adaptation to the host.

To test the hypothesis that host adaptation changes the gene expression profile, AgudeloRomero and coworkers performed an evolution experiment in which TEV was adapted to the susceptible ecotype Ler- 0 of $A$. thaliana [39•]. The TEV clone used as the ancestral virus was able to systemically infect Ler-0 plants, although the infection progressed as asymptomatic. After 17 serial undiluted passages, the resulting virus (denoted TEV-At17) fixed five point mutations, improved its accumulation by a factor of approximately three, and induced severe symptoms, including stunting, etching and leaf malformation. The set of overand underexpressed VRGs for TEV-At17 was almost three times larger. Approximately four global patterns were observed among the VRGs: genes whose expression was altered in TEV-At17-infected plants compared to plants infected with TEV; genes that were only altered after infection with the ancestral virus; genes that were only altered by the evolved virus; and genes whose expression was modified by both viruses. A search for enrichment of functional categories revealed that almost all functions downregulated by TEV were also downregulated by TEV-At17, which additionally suppresses more developmental and metabolic processes. Functions upregulated by both viruses, on the contrary, were related to plant responses to different abiotic and biotic stresses, although, interestingly, genes related to innate immune responses and response to infection were less common for TEV-At 17 than for the ancestral virus. Both viruses also differentially affected the expression of master transcription factors and highly connected proteins (results calculated from computationally inferred networks). Nevertheless, TEV-At17 regulates more central elements, in particular 41 transcription factors (vs. 26 by TEV), with more than 50 interactions (Figure 3). At face value, the above results support the hypothesis that by adapting to a host, viruses should change and improve the way they interact with the components of the host cell regulatory network.

\section{Concluding remarks}

Plants have evolved defense mechanisms to recognize pathogens and defeat them, but viruses have developed elements that interfere and suppress these mechanisms in parallel. In this review, we have addressed plant viral pathogenesis from a GSB perspective. An understanding of the mode of replication and cell interaction of viruses is an exciting question that would benefit from a global approach. The widespread use of omic techniques is leading to the emergence of new approaches towards this goal. Genome-scale network models are being predicted in silico and offer a bird's-eye view of the cell, although they could miss substantial information on punctual specific subsystems. With the aim of generating a predictive model of viral infection, these models can be used to integrate differential expression data and place VRGs into their network context. This approach would help in the design of antiviral drugs to target pathways predicted as more critical for neutralizing viral spread. Furthermore, these models could assist in the design of multidrug strategies by accounting for possible drug counteractions and undesirable effects in the cell. Overall, GSB, together with consideration of an evolutionary relationship between the virus and the plant, opens a new framework that will enable plant biologists and virologists to obtain a systematic picture for dissecting plant-virus interactions and the corresponding general and specific mechanisms, which will ultimately help to identify further agrotechnological applications. 


\section{Acknowledgements}

This work was supported by grants from the Spanish MICINN (BFU2009-06993) and Generalitat Valenciana (PROMETEO2010/019). GR is supported by a fellowship from Generalitat Valenciana (BFPI2007-160) and JC by a contract from MICINN (Grant TIN2006-12860). We thank José-Antonio Daròs and Gustavo G. Gómez for comments.

\section{References and recommended reading}

Papers of particular interest, published within the period of review, have been highlighted as:

- of special interest

-• of outstanding interest

1. Kitano H: Systems biology: a brief overview. Science 2002, 295:1662-1664.

2. • Barábasi AL, Oltvai ZN: Network biology: understanding the cell's functional organization. Nat Rev Genet 2004, 5:101-113.

In this review, the authors describe the principal network properties and the particular features that biological networks display, including hierarchy, modularity and robustness.

3. Palsson BO: In silico biology through "omics". Nat Biotechnol 2002, 20:649-650.

4. - Bansal M, Belcastro V, Ambesi-Impiombato A, di Bernardo D: How to infer gene networks from expression profiles. Mol Syst Biol 2007, 3:78.

In this review, the authors list basic guidelines for constructing large-scale network models and applying reverse-engineering techniques. In addition, they summarize the principal algorithms developed to infer regulatory networks from gene expression data.

5. Guo D, Rajamäki ML, Saarma M, Valkonen JPT: Towards a protein interaction map of potyviruses: protein interaction matrixes of two potyviruses based on the yeast twohybrid system. J Gen Virol 2001, 82:935-939.

6. - Uetz P, Dong YA, Zeretzke C, Atzler C, Baiker A, Berger B, Rajagopala SV, Roupelieva M, Rose D, Fossum E, Haas J: Herpesviral protein networks and their interaction with the human proteome. Science 2006, 311: 239-242.

This article identifies the intraviral protein interactions for two herpesviruses by yeast twohybrid assays and contextualizes them in the host interactome. The results show that whereas the viral proteins form a clustered network, the network between the viral proteins and their host targets displays scale-free properties.

7. Fossum E, Friedel CC, Rajagopala SV, Titz B, Baiker A, Schmidt T, Kraus T, Stellberger T, Rutenberg C, Suthram S, et al.: Evolutionarily conserved herpesviral protein interaction networks. PLoS Pathog 2009, 5:e1000570.

8. Lin L, Shi Y, Luo Z, Lu Y, Zheng H, Yan F, Chen J, Chen J, Adams MJ, Wu Y: Proteinprotein interactions in two potyviruses using the yeast two-hybrid system. Virus Res 2009, 142:36-40.

9. Whitham SA, Wang Y: Roles for host factors in plant viral pathogenicity. Curr Opin Plant Biol 2004, 7:365-371.

10. - Tan SL, Ganji G, Paeper B, Proll S, Katze MG: Systems biology and the host response to viral infection. Nat Biotechnol 2007, 25:1383-1389.

In this instructive commentary, the authors address the viral infection through a systems biology perspective. They show how to take advantage of omic techniques and computational methods to dissect the host-virus interaction and to design antiviral drugs.

11. Bailer SM, Haas J: Connecting viral with cellular interactomes. Curr Opin Microbiol 2009, 12:453-459.

12. Brass AL, Dykxhoorn DM, Benita Y, Yan N, Engelman A, Xavier RJ, Lieberman J, Elledge SJ: Identification of host proteins required for HIV infection through a functional genomic screen. Science 2008, 319:921-926. 
13. Krishnan MN, Ng A, Sukumaran B, Gilfoy, FD, Uchil PD, Sultana H, Brass AL, Adametz R, Tsui M, Qian F, et al.: RNA interference screen for human genes associated with West Nile virus infection. Nature 2008, 455:242-245.

14. Bushman FD, Malani N, Fernandes J, D'Orso I, Cagney G, Diamond TL, Zhou H, Hazuda DJ, Espeseth AS, König R, et al.: Host cell factors in HIV replication: meta-analysis of genome-wide studies. PLoS Pathog 2009, 5:e1000437.

15. Calderwood MA, Venkatesan K, Xing L, Chase MR, Vazquez A, Holthaus AM, Ewence AE, Li N, Hirozane-Kishikawa T, Hill DE, et al.: Epstein-Barr virus and virus human protein interaction maps. Proc Natl Acad Sci USA 2007, 104:7606-7611.

16. Dyer MD, Murali TM, Sobral BW: The landscape of human proteins interacting with viruses and other pathogens. PLoS Pathog 2008, 4:e32.

17. $\bullet$ De Chassey B, Navratil V, Tafforeau L, Hiet MS, Aublin-Gex A, Agaugué S, Meiffren G, Pradezynski F, Faria BF, Chantier T, et al:: Hepatitis $\mathbf{C}$ virus infection protein network. Mol Syst Biol 2008, 4:230.

This article identifies viral targets by yeast two-hybrid assays and shows that targeted proteins are highly connected and central in the human interactome. In addition, a functional analysis in terms of metabolic pathways is performed.

18. MacPherson JI, Dikerson JE, Pinney JW, Robertson DL: Patterns of HIV-1 protein interaction identify perturbed host-cellular subsystems. PLoS Comp Biol 2010, 6:e1000863.

19. Fregene M, Matsumura H, Akano A, Dixon A, Terauchi R: Serial analysis of gene expression (SAGE) of host-plant resistance to the cassava mosaic disease (CMD). Plant Mol Biol 2004, 56:563-571.

20. Ventelon-Debout M, Delalande F, Brizard JP, Diemer H, Van Dorsselaer A, Brugidou C: Proteome analysis of cultivar-specific deregulations of Oryza sativa indica and $\boldsymbol{O}$. sativa japonica cellular suspensions undergoing rice yellow mottle virus infection. Proteomics 2004, 4:216-225.

21. Geri C, Cecchini E, Giannakou ME, Covey SN, Milner JJ: Altered patterns of gene expression in Arabidopsis elicited by Cauliflower mosaic virus (CaMV) infection and by CaMV gene VI transgene. Mol Plant-Microbe Interact 1999, 12:377-384.

22. - Whitham SA, Quan S, Chang HS, Cooper B, Estes B, Zhu T, Wang X, Hou YM: Diverse RNA viruses elicit the expression of common sets of genes in susceptible Arabidopsis thaliana plants. Plant J 2003, 33:271-283.

This article analyzes a compendium of gene expression profiles in plants for the first time to compare the infection mechanisms of different viruses in a common host, and highlights some generalities.

23. Golem $\mathrm{S}$, Culver JN: Tobacco mosaic virus induced alterations in the gene expression profile of Arabidopsis thaliana. Mol Plant-Microb Interact 2003, 16:681-688.

24. Espinoza C, Medina C, Somerville S, Arce-Jonhson P: Senescence-associated genes induced during compatible viral interactions with grapevine and Arabidopsis. $J$ Exp Bot 2007, 58:3197-3212.

25. Ishihara T, Sakurai N, Sekine KT, Hase S, Ikegami M, Shibata D, Takahashi H: Comparative analysis of expressed sequence tags in resistant and susceptible ecotypes of Arabidopsis thaliana infected with cucumber mosaic virus. Plant Cell Physiol 2004, 45:470-480.

26. Marathe R, Guan Z, Anandalakshmi R, Zhao H, Dinesh-Kumar SP: Study of Arabidopsis thaliana resistome in response to cucumber mosaic virus infection using whole genome microarray. Plant Mol Biol 2004, 55:501-520. 
27. Yang C, Guo R, Jie F, Nettleton D, Peng J, Carr T, Yeakely JM, Fan JB, Whitham SA: Spatial analysis of Arabidopsis thaliana gene expression in response to turnip mosaic virus infection. Mol Plant-Microb Interact 2007, 20:358-370.

28. Babu M, Griffiths JS, Huang TS, Wang A: Altered gene expression changes in Arabidopsis leaf tissues and protoplasts in response to plum pox virus infection. $B M C$ Genomics 2008, 9:325.

29. Agudelo-Romero P, Carbonell P, De la Iglesia F, Carrera J, Rodrigo G, Jaramillo A, Pérez-Amador MA, Elena SF: Changes in the gene expression profile of Arabidopsis thaliana after infection with tobacco etch virus. Virol J 2008, 5:92.

30. Trinks D, Rajeswaran R, Shivaprasad PV, Akbergenov R, Oakeley EJ, Veluthambi K, Hohn T, Pooggin MM: Suppression of RNA silencing by a geminivirus nuclear protein, AC2, correlates with transactivation of host genes. J Virol 2005, 79:25172527.

31. Ascencio-Ibáñez JT, Sozzani R, Lee TJ, Chu TM, Wolfinger RD, Cella R, HanleyBowdoin L: Global analysis of Arabidopsis gene expression uncovers a complex array of changes impacting pathogen response and cell cycle during geminivirus infection. Plant Physiol 2008, 148:436-454.

32. Wise RP, Moscou MJ, Bogdanove AJ, Whitham SA: Transcript profiling in hostpathogen interactions. Annu Rev Phytopathol 2007, 45:329-369.

33. Jockusch H, Wiegand C, Mersch B, Rajes D: Mutants of tobacco mosaic virus with temperature-sensitive coat proteins induce heat shock response in tobacco leaves. Mol Plant-Microb Interact 2001, 14:914-917.

34. De la Fuente A: From 'differential expression' to 'differential networking' identification of dysfunctional regulatory networks in diseases. Trends Genet 2010, 26:326-333.

35. Warsow G, Greber B, Falk SS, Harder C, Siatkowski M, Schordan S, Som A, Endlich N, Scholer H, Repsilber D, et al:: ExprEssence - revealing the essence of differential experimental data in the context of an interaction/regulation network. BMC Syst Biol 2010, 4:164.

36. Geisler-Lee J, O’Toole N. Ammar R, Provart NJ, Millar AH, Geisler M: A predicted interactome for Arabidopsis thaliana. Plant Physiol 2007, 145:317-329.

37. Ma S, Gong Q, Bohnert HJ: An Arabidopsis gene network based on the graphical Gaussian model. Genome Res 2007, 17:1614-1625.

38. Carrera J, Rodrigo G, Jaramillo A, Elena SF: Reverse-engineering the Arabidopsis thaliana transcriptional network under changing environmental conditions. Genome Biol 2009, 10:R96.

39. - Agudelo-Romero P, Carbonell P, Pérez-Amador MA, Elena SF: Virus adaptation by manipulation of host's gene expression. PLOS ONE 2008, 3:e2397.

This article reports results for an evolution experiment of TEV on A. thaliana in which the evolved virus triggers a different plant transcriptomic pattern compared to the ancestral one.

40. Pagán I, Fraile A, Fernández-Fueyo E, Montes N, Alonso-Blanco C, García-Arenal F: Arabidopsis thaliana as a model for the study of plant-virus coevolution. Phil Trans $R$ Soc B 2010, 365:1983-1995.

Figure 1. List of biological functions up- and downregulated by plant viruses in A. thaliana.

Figure 2. Connectivity distribution for the whole plant interactome (red line) and the distribution generated by the VRGs (green line) for two opposing modes of virus action. Panel (a) illustrates the case of VRGs being sparsely distributed in the network and poorly 
connected. This situation translates into connectivity distributions steeper than observed the whole interactome. Panel (b) exemplifies the opposite situation of VRGs being highly connected hubs. In this case the connectivity distribution is flatter

Figure 3. Number of (a) master transcription factors and (b) hub proteins altered by infection with wild type TEV (blue bars) and with $A$.thaliana adapted TEV-At17 (red bars). See main text or ref. [39•] for details about these two isolates.

Table 1 Number of virus-regulated genes and interactions and degree of connectivity $\langle k\rangle$ for different viruses for the $A$. thaliana transcriptional regulatory network (TRN) and proteinprotein interaction network (PPIN).

\begin{tabular}{|c|c|c|c|c|c|}
\hline Virus & VRGs $^{\mathrm{a}}$ & Interactions $^{\mathrm{TRN}}$ & $\langle k\rangle^{\mathrm{TRN}}$ & Interactions $^{\text {PPIN }}$ & $\langle k\rangle^{\text {PPIN }}$ \\
\hline \multicolumn{6}{|c|}{ Viruses naturally infecting Brassicaceae } \\
\hline $\mathrm{CaLCV}$ & 1186 & 2108 & 255 & 664 & 24 \\
\hline $\mathrm{TCV}$ & 1554 & 4326 & 188 & 364 & 19 \\
\hline TEV-At 17 & 2391 & 2840 & 115 & 881 & 22 \\
\hline TuMV & 1144 & 1026 & 172 & 1665 & 34 \\
\hline Average & 1568.75 & 2575.00 & 182.50 & 893.50 & 24.75 \\
\hline \multicolumn{6}{|c|}{ Viruses not naturally infecting Brassicaceae } \\
\hline PPV & 1487 & 939 & 153 & 535 & 24 \\
\hline TEV & 678 & 1269 & 162 & 64 & 18 \\
\hline TMV & 723 & 67 & 76 & 214 & 22 \\
\hline TRV & 499 & 82 & 111 & 154 & 26 \\
\hline Average & 846.75 & 589.25 & 125.50 & 241.75 & 22.50 \\
\hline$P^{\mathrm{b}}$ & 0.047 & 0.020 & 0.077 & 0.035 & 0.282 \\
\hline
\end{tabular}

${ }^{a}$ Virus-regulated genes identified by differential expression in microarray data comparing mock-inoculated plants and plants infected by the corresponding virus.

${ }^{\mathrm{b}}$ Statistical significance assessed by one-tailed $t$-tests. 